\title{
The use of non-invasive ventilation for acute respiratory failure in general medical wards: a regional Italian survey
}

\author{
Federico Lari, ${ }^{1}$ Fabio Gilioli, ${ }^{2}$ Maurizio Ongari, ${ }^{3}$ Giorgio Ballardini ${ }^{4}$
}

${ }^{1}$ UOC Medicina Interna, Ospedale di San Giovanni in Persiceto, AUSL Bologna; ${ }^{2}$ UOC Medicina Interna, Ospedale di Mirandola, AUSL Modena; ${ }^{3}$ UOC Medicina Interna, Ospedale di Porretta Terme, AUSL Bologna; ${ }^{4}$ UOC Medicina Interna, Ospedale di Rimini, AUSL Romagna, Italy

\begin{abstract}
Non-invasive ventilation (NIV) is a rapidly spreading method in the last years even outside of intensive care units for the treatment of patients with acute respiratory failure. Its use in general medical wards in Italy and Europe is still largely incomplete and there are clear gaps in terms of organization, training, selection, and patient monitoring. If these gaps are filled, NIV has also proven to be effective in general medical wards, especially if they have a critical care area. This publication reports the data collected by an Italian regional survey on the use of NIV in internal medicine, highlighting positive and negative aspects.
\end{abstract}

\section{Introduction}

Non-invasive mechanical ventilation (NIV) is a rapidly spreading method in the last years for the treatment of various forms of acute respiratory failure (ARF). Under certain clinical conditions, it has proven to be effective in reducing the need for intubation, acute mortality, intensive care stay time, costs related to patient hospitalization, as well as in improving clinical and gas-analytic parameters. ${ }^{1-3}$ Developed first within intensive care units in the hands of physicians to try to reduce complications related to tracheal intubation and invasive mechanical ventilation, its use has subsequently expanded into other areas, with documented effectiveness, between the emergency departments and general medical wards due to various

Correspondence: Federico Lari, UOC Medicina Interna, Ospedale di San Giovanni in Persiceto (BO), AUSL Bologna, via Enzo Palma 1, 40017 San Giovanni in Persiceto (BO), Italy. E-mail: federico.lari@ausl.bologna.it

Key words: Acute respiratory failure; general medical ward; non-invasive ventilation.

Conflict of interests: the authors declare no potential conflict of interests.

Received for publication: 18 January 2020.

Accepted for publication: 27 February 2020.

This work is licensed under a Creative Commons Attribution NonCommercial 4.0 License (CC BY-NC 4.0).

${ }^{\circ}$ Copyright: the Author(s), 2020

Licensee PAGEPress, Italy

Italian Journal of Medicine 2020; 14:62-68

doi:10.4081/itjm.2020.1260 factors: ${ }^{4-7}$ i) the need to treat patients with ARF early to avoid deterioration of respiratory function; ii) the shortage of beds in intensive care; iii) the increasing number of elderly, fragile, immunocompromised patients, with comorbidities that are not eligible for intubation and therefore manageable outside intensive care units; iv) the growing evidence of the effectiveness of the method also in general medical wards if organization and logistics, training of dedicated staff, accurate patient selection, and adequate monitoring are present; v) evidence of the effectiveness of NIV also for palliative care, for symptom control.

The problem of setting where to make NIV has been debated for years in literature. The etiology and severity of ARF certainly play an important role in the choice but today also the changed epidemiology of patients, age and comorbidity, are decisive..$^{7-10}$ Lately, there were no documents of international consensus that defined the minimum criteria necessary to activate a NIV service within a hospital; today, however, the UK guidelines ${ }^{2,4}$ define this area as level 2 of intensive care, a facility in which there is at least one dedicated nurse every 2 patients in NIV (during daylight hours), and where are managed patients with organ failure with appropriate methods to support it, according to the philosophy of non-invasiveness, with a valid local organization. These characteristics are well suited to the critical/semi-intensive areas born in recent years within some Units of Internal Medicine in Italy according to organizational models for intensity of care and care complexity, but also where these areas are not well structured the literature allows the use of NIV in internal medicine departments if organizational, logistical and training standards are respected. ${ }^{5}$

Despite this, the current use of NIV during ARF in medical units in Italy is still very heterogeneous. ${ }^{11}$ 
There are places where the method is well known and used with a good organization, while there are other realities where the ventilator is not present and the management of these patients is always and in any case referred to other specialists; between these 2 extremes there are intermediate situations very different from each other although geographically close, sometimes also belonging to the same region. Surveys prior to this ${ }^{12-16}$ showed that the main limitations related to the use of NIV in internal medicine relate to gaps in organization, training and resources. ${ }^{17-23}$

\section{Materials and Methods}

FADOI (Federation of Associations of Hospital Doctors on Internal Medicine) is a scientific society of Italian hospital internist physicians very active in the field of training and research, organized in autonomous regional directors and a central national organization. In 2018, the Emilia Romagna section decided to draw up a questionnaire on the use of NIV in ARF to be submitted to the units of internal medicine of the region to collect information on how and if they managed NIV. The questionnaire included 23 multiple-choice questions (see online Appendix 1 - in Italian), it was easy to understand and quickly filled in (a text file with pre-filled form fields was used), it was sent by e-mail to the directors of the internal medicine units of the Emilia Romagna region. 81 units were contacted, 33 responded by resubmitting the completed questionnaire. Collected data were transferred to an electronic spreadsheet in which statistical processing was carried out. The surveyed units did not present a specific pneumological vocation, only in 2 out of 33 cases there was a pulmonologist in the staff.

\section{Results}

The 33 units that responded to the survey were located in 30 different hospitals. Table 1 shows the percentage of units within the hospital based on the number of beds. Most of the units that responded to the questionnaire were therefore placed in small hospitals.

Table 2 shows the units that have joined the survey according to the province they belong to.

The estimated percentage of patients treated with NIV for ARF in one year was $7.36 \%$ of admissions, the average value of patients treated with NIV was $7.36 \%$ of hospitalizations with a range from 1 to $20 \%$ in the various centers. Out of the 33 centers that responded to the survey, only 1 stated that they did not use any NIV methods: it is clear that this report, in favor of centers using NIV, could be overestimated by the fact that these centers, already confident about the method, were more motivated to respond.
With regard to ventilation techniques and technologies: i) CPAP was used by $87 \%$ of the centers, $92 \%$ owned a CPAP device in the unit, usually a simple Venturi flow generator or a disposable system; ii) double-pressure methods (bilevel or pressure support ventilation) were used in $83 \%$ of the centers. Fifty-one percent of operating units said they had at least one ventilator (range 1-4), and 32\% said they would use it on loan from other units if necessary. In $88 \%$ of cases ventilators consisted in simple, home-derived machines or NIV-specific ventilators; no centers used complex intensive care ventilators.

Table 3 shows the interfaces used by the different centers.

The average experience of using NIV was overall 11 years for CPAP (range 4-20), and 9 years for bilevel-pressure methods (range 3-18).

The main forms of acute or acute on chronic respiratory failure treated with NIV in the different centers are shown in Table 4. COPD exacerbation with

Table 1. Percentage of internal medicine units that joined the study based on the size of the hospital in which they were placed.

\begin{tabular}{lc}
\hline$<150$ B. & $40 \%$ \\
\hline $151-250$ B. & $16.6 \%$ \\
\hline $251-500$ B. & $20 \%$ \\
\hline$>501$ B. & $23.3 \%$ \\
\hline
\end{tabular}

B., beds.

Table 2. Number of internal medicine units who joined the study based on the province they belong to in Emilia Romagna.

\begin{tabular}{lc}
\hline Bologna & 11 units \\
\hline Modena & 9 units \\
\hline Reggio Emilia & 3 units \\
\hline Ravenna & 1 unit \\
\hline Rimini & 5 units \\
\hline Forlì Cesena & 1 unit \\
\hline Parma & 2 units \\
\hline Piacenza & 1 unit \\
\hline
\end{tabular}

Table 3. Types of interfaces used and their prevalence.

\begin{tabular}{lc}
\hline Facial Mask & $100 \%$ \\
\hline Total Face & $20 \%$ \\
\hline Nasal Mask & $10 \%$ \\
\hline Helmet & $3.3 \%$
\end{tabular}


respiratory acidosis and acute cardiogenic pulmonary edema, were treated in all centers. These are the forms of ARF that are most prominent in terms of effectiveness of NIV even outside of intensive care.

In terms of local organization, only $35 \%$ of units had a well-defined and structured critical/semi-intensive area within the ward where they could manage patients on NIV. In $65 \%$ of cases, therefore, patients were ventilated in traditional general medical ward. Diagnostic and therapeutic path on management of ARF were present in only $35 \%$ of the centers, and in this case the internist was not involved in the design of the path that involved other specialists (emergency, resuscitators, pneumologists) instead.

Among the medical staff of the units that implemented NIV, there was an expert internal physician in only $62 \%$ of cases. The prescription of ventilator treatment and monitoring was carried out independently by the internists of the ward only in a minority of cases: more often the treatment consisted in a co-management with other specialists (Table 5).

The presence of protocols within the medical unit or hospital for the management of patients with ARF on NIV was present in only $46 \%$ of cases; these protocols were complete in their essential parts as defined by the guidelines only in $20 \%$ of cases. Table 6 shows the single components of the protocols in relation to the percentage of centers that covered them.
With regard to patient monitoring during ventilator treatment, this was completed, according to the guidelines, only in $13 \%$ of cases. $40 \%$ of medical units reported the use of less than 4 parameters. Table 7 shows the different monitoring parameters indicated as essential by the literature in relation to the percentages of the centers that used them.

The NIV Team provides for the involvement of all specialists of a single hospital dedicated to the management of the patient with ARF on NIV, who must necessarily speak the same language, be trained in a homogeneous way, in order to give continuity to the treatment of the patient. Depending on the size of the hospital the following healthcare professionals may be involved: emergency physicians, resuscitators, pneumologists, internists, cardiologists, and others. Its function, essential for the effectiveness of treatment, has been defined by the guidelines for years. In our survey, the NIV team was present in only $21 \%$ of NIV practice centers, in no hospital there were defined network models such as $H u b \&$ Spoke for example for the rapid centralization of the most critical patients or on the contrary for the reliance on the hospital of territorial competence for stabilized patients. In most cases there was no therapeutic continuity even with regard to the technologies used (masks, circuits, different ventilators).

The most common complications related to NIV

Table 4. Types of acute respiratory failure and their prevalence in different medical units.

\begin{tabular}{|c|c|}
\hline Acute exacerbation of chronic obstructive pulmonary disease & $100 \%$ \\
\hline Acute cardiogenic pulmonary edema & $100 \%$ \\
\hline Pneumonia & $52 \%$ \\
\hline Inhalation & $46 \%$ \\
\hline Immunodepressed & $41 \%$ \\
\hline DNI (patient not to be intubated) & $39 \%$ \\
\hline neuromuscular disease & $33 \%$ \\
\hline Acute respiratory distress syndrome & $12 \%$ \\
\hline Asthma & $9 \%$ \\
\hline
\end{tabular}

Table 5. Prescriptive and non-invasive ventilation treatment monitoring responsibilities in the different centers involved in the survey in relation to the different specialists involved.

\begin{tabular}{llll}
\hline & & Autonomy & Co-management \\
\hline CPAP & $\mathbf{8 8 . 5} \%$ Internist & $\mathbf{5 1 . 5 \%}$ & $22 \%$ Anesthesiologist \\
\hline $7.5 \%$ Pneumologist & & $11 \%$ Pneumologist \\
\hline Bi-level & $4 \%$ Anesthesiologist & & $4 \%$ Emergency Physician \\
\hline & $\mathbf{6 5 \%}$ Internist & $\mathbf{1 9 \%}$ & $24 \%$ Anesthesiologist \\
\hline & $15 \%$ Pneumologist & & $18 \%$ Pneumologist \\
\hline
\end{tabular}


treatment and their overall prevalence in our survey are shown in Table $8.40 \%$ of medical units report more than 4 frequent complications. As you can see, it is a higher prevalence than the data reported in lit-

Table 6. Aspects covered in non-invasive ventilation management protocols and their prevalence in the different medical units.

\begin{tabular}{ll}
\hline Patient selection & $100 \%$ \\
\hline Indications/Contraindications & $86 \%$ \\
\hline Ventilation mode & $86 \%$ \\
\hline Prescription therapy card & $46 \%$ \\
\hline Definition of the non-invasive ventilation trial & $40 \%$ \\
\hline Definition of failure & $73 \%$ \\
\hline Monitoring & $73 \%$ \\
\hline Weaning & $46 \%$ \\
\hline
\end{tabular}

Table 7. Monitoring parameters and their prevalence in the different medical units.

\begin{tabular}{ll}
\hline Continuous electrocardiogram (pt with arrhythmia) & $40 \%$ \\
\hline $\mathrm{SpO}_{2}$ continuously & $60 \%$ \\
\hline Arterial blood pressure & $68 \%$ \\
\hline Heart rate & $68 \%$ \\
\hline Respiratory rate & $64 \%$ \\
\hline Neurological score & $32 \%$ \\
\hline EGA & $100 \%$ \\
\hline Nursing assessment & $92 \%$ \\
\hline Medical evaluation & $92 \%$ \\
\hline
\end{tabular}

Table 8. Prevalence of non-invasive ventilation complications most frequently reported by the different medical units in the study.

\begin{tabular}{lc}
\hline Mask pressure injuries & $68 \%$ \\
\hline Excessive agitation & $52 \%$ \\
\hline Excessive air leaks & $40 \%$ \\
\hline ventilator malfunction & $8 \%$ \\
\hline Absolute rejection of treatment & $36 \%$ \\
\hline Poor collaboration of nursing staff & $4 \%$ \\
\hline Risk of inhalation/vomiting & $12 \%$ \\
\hline Risk of sinusitis/conjunctivitis & $4 \%$ \\
\hline Mucous dryness & $36 \%$ \\
\hline Claustrophobia & $8 \%$ \\
\hline Insomnia & $12 \%$ \\
\hline Risk of delaying intubation & $12 \%$ \\
\hline Gastric détente & $4 \%$ \\
\hline
\end{tabular}

erature in other settings; this can be interpreted as a lack of local organization and staff training.

The involvement of nursing staff is essential, therefore, staff training has always been regarded by literature as one of the secrets of NIV success; it is fundamental to ensure a successful treatment, and also in terms of motivational aspects. In detail: i) training at the beginning of the center NIV experience: $15 \%$ of the centers did not complete initial training, $60 \%$ carried it out within the hospital, only $25 \%$ even with events outside the hospital; ii) periodical Retraining on a minimum annual basis: only $36 \%$ of units have been reported; iii) the involvement of nursing staff was considered optimal by $75 \%$ of the centers surveyed; iv) the feeling, the awareness of the effectiveness of NIV is still considered high, $100 \%$ of the physicians who answered the questionnaire have this opinion.

\section{Discussion}

The data collected in this Italian regional survey are essentially in line with what is reported in the literature in similar surveys conducted in recent years both in Italy and abroad, ${ }^{11-23}$ with the particularity that this study, unlike others, is conducted exclusively in medical units that did not have a specific pneumological address: the NIV is rapidly spreading in internal medicine but still with non-homogeneous distribution even in limited geographical areas. This is often due to the lack of structured pathways for the patient with ARF by hospitals, and the management of these clinical conditions is thus left to the spontaneous initiative of the individual centers and to the local organization. In many internal medicine realities, despite the enthusiasm towards the method, there are still obvious gaps in terms of organization and logistics.

Interestingly, the autonomy of prescription and management of treatment with NIV, as well as the ownership of ventilators by the medical unit, the percentage of patients treated and a better organization were inversely related to the size of the hospital in which the medical ward was located. This makes, at least in Emilia Romagna, small and peripheral hospitals generally more experienced and autonomous in managing NIV; this is easily understood if we consider the role of internal medicine outside the large centers, which must often include the emergency medicine or at least supply the lack of skills not present in the hospital. On the other hand, in internal medicine wards located in large centers, NIV is less widespread and the team's skills are not adequately developed; this because, in large hospitals, there are other specialists involved in the management of the patient with ARF, often in intensive care setting (generalist or specialist intensive care units, emergency medicine, step down 
units) and if the patient on NIV is managed in a medical ward, the internist is not the central figure but the other specialist as consultants (pulmonologist, resuscitator, emergency physician). Of the 7 internal medicine units located in hospitals with more than 500 beds participating in the study, 3 do not practice Bilevel ventilation but only CPAP occasionally, 3 practice Bilevel ventilation but do not have their own ventilators, therefore the ventilator is brought to the ward in case of need by the specialist involved in the prescription of the treatment (typically anesthesiologist or pneumologist). A medical unit finally stated that it did not practice any NIV methods. In most cases these medical units are located in University Hospitals where, in case of need of NIV, the patient is preferably transferred to other structures (usually intensive care). This particular situation highlights the problem of training on these issues of young internists who are often not so satisfied with the educational needs on topics of absolute relevance for modern internal medicine.

Another very important fact that emerges from this survey is that even the centers considered the most expert in NIV and that treat a large number of patients have obvious gaps in terms of organization and logistics. Doing NIV out of a protected environment such as intensive care or step-down unit and especially in general medicine wards where the personal/patient relationship is unfavorable imposes a high-level organization. Although guidelines ${ }^{2}$ suggest we preferentially manage these patients within critical areas, this is still not possible in all internal medicine units: the literature also tells us that many of the studies conducted in wards without critical areas ${ }^{10,24-28}$ showed NIV success rates similar to those reported in other studies conducted in intensive care units, if proper patient selection has been implemented and high organizational and safety standards are guaranteed. It is therefore necessary to implement aspects of the organization and management of treatment in the medical department such as: i) the presence of a physician more expert that acts as coordinator of the working group, granting his/her availability for the discussion of the most critical cases; ii) facilitating the development of therapeutic diagnostic pathways and clinical networks on the Hub \& Spoke model within the hospitals for the rapid centralization and easy access to intensive care for critical patients; iii) to promote the establishment of multidisciplinary and multi-professional teams dedicated to patient management during ARF in NIV within the hospital. Not only the different medical specialists but also the nurses whose role, in the success of this method, is undisputed; iv) implement the development of internal protocols in the medical unit covering the different aspects related to treatment with NIV: correct patient selection, indications/contraindications, setting of ventilator parameters, pre- scription card of treatment, definition of trial and failure, elaboration of an individualized care plan for each patient, weaning techniques; v) improve and refine patient monitoring in NIV, which must not be particularly aggressive and complex, but respond to the philosophy of non-invasiveness and simplicity, and at the same time it must be precise and timely. Standalone bedside multi-parameter monitoring systems but with transmission of data and alarms to control unit and portable systems (tablets) are well suited to the structure of a general medical ward without necessarily forcing the design of a critical area. Limiting invasiveness as much as possible, in this perspective the assessment of blood gases can also be carried out on capillary sampling, $\mathrm{CO}_{2}$ can also be monitored by transcutaneous way $\left(\mathrm{TcPCO}_{2}\right) ;{ }^{2}$ vi) to facilitate the adaptation of the patient to treatment and synchrony with the ventilator, through the choice of a correct interface, proper nursing, the adoption of protocols and sedation techniques, a correct setting of ventilation parameters, minimizing complications and side effects.

Finally, another aspect to be refined within the operators who devote themselves to the NIV in internal medicine is the specific training, which, as evidenced by the data of this survey, is often lacking. Staff must be trained not only at the beginning of their experience with NIV but must also be trained periodically with moments of refresher courses that involve practical aspects. Healthcare facilities should be encouraged to organize internal training and clinical audits on these issues, but they should also make use of high-quality external training events (scientific societies or other companies with documented experience in the field). The recent and well-established turnover of medical and nursing staff in internal medicine requires further attention to continuous and periodic training.

In conclusion, we believe that some final considerations regarding the use of NIV in internal medicine are necessary, which emerge spontaneously from the data of this survey. The changing epidemiology of medical patients today, which includes elderly, complex, fragile and comorbidity patients, together with the lack of beds in intensive care units, has meant that, as needed, internal medicine approached the NIV: a large proportion of patients with ARF are managed in general medicine wards and in any case outside of intensive care because they would not be eligible for intubation and invasive ventilation (sometimes also by express will), and this trend is likely to increase in the next years especially for those patients with ARF potentially responsive to NIV (e.g. COPD exacerbated with respiratory acidosis, acute cardiogenic pulmonary edema).

While large randomized controlled trials on the effectiveness of NIV in the different causes of ARF ${ }^{29,30}$ have always enrolled relatively young patients without 
relevant comorbidities trying to demonstrate important endpoints (such as reduction in the need for intubation and mortality), today observational data from the use of NIV in the real world ${ }^{4,31-33}$ tell us that we often treat completely different patients (who by characteristic and severity would have been excluded from large trials) and with different goals and results: in these patients NIV should aim to reduce symptoms (breathlessness), to correct gas exchanges, to overcome the acute event, well aware that the survival of the patient often depends on age and other chronic clinical conditions with unfavorable prognosis. Here too the role of internal medicine is inevitable, palliative treatment of patients with end-stage pathologies, not only cancer, is now an important part of the daily work of the internist, and the literature tells us that NIV can play an important role. ${ }^{3}$

Today, in patients with ARF, the choice of respiratory support, non-invasive versus invasive, represents a crucial factor, not only in terms of severity and etiology of the ARF (probability of favorable response to NIV) but also in terms of patient's characteristics (age, comorbidity, will) and of organization/local resources.

\section{Conclusions}

NIV in ARF is also effective in general medical wards if certain fundamental rules are respected, such as: i) the correct selection of patients; ii) the knowledge that it is not an alternative to invasive ventilation if this is indicated; iii) early application and proper monitoring; iv) high standards for organization, training and logistics.

The survey conducted by FADOI Emilia Romagna in the internal medicine units of the region showed that: i) bi-level pressumetric ventilation methods are less widely available globally but clearly prevalent in small peripheral hospitals; ii) CPAP is more widespread in general and for longer; iii) small centers are more experienced, organized and autonomous; iii) comanagement with other specialists is frequent, especially in large centers; iv) treatment setting: only $35 \%$ ventilate in critical area, $65 \%$ in traditional ward; v) only half of the centers have their own ventilators, generally simple of home derivation; vi) the experience on NIV is not high, limited in time, but there is enthusiasm and desire to learn; vii) predominantly treated forms of ARF are: exacerbation of COPD, acute cardiogenic pulmonary edema; viii) there are important gaps in terms of staff organization and training: internal protocols, NIV team, expert internal physician, therapeutic diagnostic pathways, Hub \& Spoke models, monitoring, involvement of nursing staff, basic training and periodic retraining.

For an effective NIV treatment it is important how you do it, not where. In order to further spread these methods in Italian internal medicine units, it is desirable to try to further homogenize different realities and filling the shortcomings currently present. In this direction, the role of scientific societies, which have always been involved in research and training, can be of great importance.

\section{References}

1. British Thoracic Society Guidelines. Non-invasive ventilation in acute respiratory failure. Thorax 2002;57:1 92-211.

2. Davidson AC, Banham S, Elliott M, et al. BTS/ICS guideline for the ventilatory management of acute hypercapnic respiratory failure in adults. Thorax 2016;71:ii1-ii35.

3. Rochwerg B, Brochard L, Elliott M, et al. Official ERS/ATS clinical practice guidelines: noninvasive ventilation for acute respiratory failure. Eur Respir J 2017;50:1-20.

4. Bourke SC, Piraino T, Pisani L, et al. Beyond the guidelines for non-invasive ventilation in acute respiratory failure: implications for practice. Lancet Respir Med 2018;6:935-47.

5. Davies M, Allen M, Bentley A, et al. British Thoracic Society Quality Standards for acute non-invasive ventilation in adults. BMJ Open Resp Res 2018;5:e000283.

6. Keenan SP, Sinuff T, Burns KEA, et al. Clinical practice guidelines for the use of noninvasive positive-pressure ventilation and noninvasive continuous positive airway pressure in the acute care setting. CMAJ 2011;183:E195214.

7. Navalesi P, Campanini M, Lari F, et al. La ventilazione non invasiva in medicina interna. Quad Ital $\mathrm{J}$ Med 2015;3(5):391-498.

8. Lari F, Giostra F, Bragagni G, et al. La ventilazione meccanica non invasiva nell'insufficienza respiratoria acuta: stato dell'arte (Parte I). Ital J Med 2009;3:201-11.

9. Lari F, Giostra F, Bragagni G, et al. La ventilazione meccanica non invasiva nell'insufficienza respiratoria acuta: stato dell'arte (Parte II). Ital J Med 2010;4:6-15.

10. Lari F, Scandellari N, DeMaria F, et al. La ventilazione meccanica non invasiva nel trattamento dell'insufficienza respiratoria acuta: efficacia ed applicabilità in reparto medico. Emerg Care J 2009;5:8-20.

11. Cabrini L, Antonelli M, Savoia G, Landriscina M. Noninvasive ventilation outside of the intensive care unit: an Italian survey. Minerva Anestesiol 2011;77:313-22.

12. Cabrini L, Esquinas A, Pasin L, et al. An international survey on non-invasive ventilation use for acute respiratory failure in general non-monitored wards. Respir Care 2015;60:586-92.

13. Bierer GB, Soo Hoo GW. Noninvasive ventilation for acute respiratory failure: a national survey of Veterans Affair Hospitals. Respir Care 2009;54:1313-20.

14. Guarracino F, Cabrini L, Ferro B, et al. Noninvasive ventilation practice in cardiac surgery patients: insights from a European survey. J Cardiothorac Vasc Anesth 2013;27:e63-e65.

15. Burns KE, Sinuff T, Adhikari NK, et al. Bilevel positive pressure ventilation for acute respiratory failure: survey 
of Ontario practice. Crit Care Med 2005;33:1477-83.

16. Doherty MJ, Greenstone MA. Survey of non-invasive ventilation in patients with acute exacerbations of chronic obstructive pulmonary disease in the UK. Tho$\operatorname{rax}$ 1998;53:863-6.

17. Kumle B, Haisch G, Suttner SW, et al. Current status of non-invasive ventilation in German ICUs: a postal survey. Anasthesiol Intensiv Med 2003;38:32-37.

18. Crimi C, Noto A, Princi P, et al. A European survey of non-invasive ventilation (NIV) practices. Eur Respir J 2010;36:362-9.

19. Demoule A, Girou E, Richard JC, et al. Increased use of noninvasive ventilation in French intensive care units. Intensive Care Med 2006;32:1747-55.

20. Hess DR, Pang JM, Camargo CA Jr. A survey of the use of noninvasive ventilation in academic emergency departments in the United States. Respir Care 2009;54:1306-12.

21. Maheshwari V, Paioli D, Rothaar R, et al. Utilization of noninvasive ventilation in acute care hospitals: a regional survey. Chest 2006;129:1226-33.

22. Scala R, Windisch W, Kohnlein T, et al. Targeting European Respiratory Society Group activities: a survey of the Noninvasive Ventilatory Support Group. Eur Respir Rev 2014;23:258-60.

23. Scala R, Corrado A, Confalonieri M, et al. Increased number and expertise of Italian respiratory high-dependency care units: the second national survey. Respir Care 2011;56:1100-7.

24. Carlucci A, Delmastro M, Rubini F, et al. Changes in the practice of non-invasive ventilation in treating COPD patients over 8 y. Intensive Care Med 2003;29:419-25.

25. Plant PK, Owen JL, Parrott S, Elliott MW. Cost effectiveness of ward based non-invasive ventilation for acute exacerbations of chronic obstructive pulmonary disease: economic analysis of randomised controlled trial. BMJ 2003;326:956-61.

26. Plant PK, Owen JL, Eilliott MW. Early use of Non-invasive ventilation (NIV) in acute exacerbations of COPD on general respiratory ward: a multicentre randomised controlled trial. Lancet 2000;355:1931-5.

27. Barbe F, Togores B, Rubi M, et al. Noninvasive ventilatory support does not facilitate recovery from acute respiratory failure in chronic obstructive pulmonary disease. Eur Respir J 1996;9:1240-5.

28. Bott J, Carroll MP, Conway JH, et al . Randomized controlled trial of nasal ventilation in acute ventilatory failure due to chronic obstructive airways disease. Lancet 1993;341:1555-7.

29. Plant PK, Owen JL, Elliott MW. Non-invasive ventilation in acute exacerbations of chronic obstructive pulmonary disease: long term survival and predictors of in-hospital outcome. Thorax 2001;56:708-12.

30. Hess DR. Non invasive ventilation for acute respiratory failure. Respir Care 2013;58:950-72.

31. British Thoracic Society. BTS audit reports 2010 to 2013. British Thoracic Society; 2018. Available from: https:// www.brit-thoracic.org.uk/publication-library/bts-reports/

32. National Review of Asthma Deaths. Why asthma still kills. Royal College of Physicians, 2014. Available from: https://www.rcplondon.ac.uk/projects/outputs/whyasthma-still-kills

33. Chandra D, Stamm JA, Taylor B, et al. Outcomes of noninvasive ventilation for acute exacerbations of chronic obstructive pulmonary disease in the United States, 1998-2008. Am J Respir Crit Care Med 2012;185:152-9. 